\title{
Liver Abscess Caused by Fusobacterium nucleatum: A Case Report
}

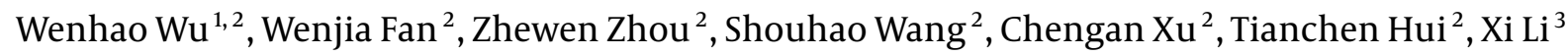 \\ and Hongying $\operatorname{Pan}^{2, *}$ \\ ${ }^{1}$ Medical College, Qingdao University, Qingdao, China \\ ${ }^{2}$ Department of Infectious Diseases, Zhejiang Provincial People's Hospital, Hangzhou, China \\ ${ }^{3}$ Centre of Laboratory Medicine, Zhejiang Provincial People's Hospital, Hangzhou, China \\ "Corresponding author: Department of Infectious Diseases, Zhejiang Provincial People’s Hospital, Hangzhou, China. Email: hypanzjsrmyy@126.com \\ Received 2021 May 29; Revised 2021 June 15; Accepted 2021 June 15.
}

\begin{abstract}
Introduction: Pyogenic liver abscess (PLA) is a serious infectious disease of the liver. Pyogenic liver abscess caused by Fusobacterium nucleatum is extremely rare. Here we report the first case of liver abscess caused by F. nucleatum in China.

Case Presentation: The case was a 34-year-old female patient admitted to the hospital due to high fever. The diagnosis of liver abscess was confirmed by imaging studies and liver puncture. We finally confirmed the pathogen as F.nucleatum by next-generation sequencing (NGS). After the targeted anti-infective treatment, the patient recovered and discharged.

Conclusions: As a new microbial detection method, NGS can still help in clinical practice. In addition, to improve the positive rate of anaerobic bacteria culture, we should pay attention to avoid contact with air in the process of specimen collection when the pathogenic bacteria are suspected to be anaerobic bacteria.
\end{abstract}

Keywords: Pyogenic liver abscess, Fusobacterium nucleatum, Next-Generation Sequencing, Immunocompetent

\section{Introduction}

Pyogenic liver abscess (PLA) is caused by pyogenic bacteria invading the liver. The incidence rate of liver abscess in the United States is about 4.1/100000, while in Asian countries is about $11.99 \sim 17.59 / 100000(1,2)$. The mortality of bacterial liver abscess is very high. A study of 431 patients in Taiwan showed that the mortality rate was as high as $15 \%$ (3). The pathogens enter the liver mainly in the following ways: (1) biliary infection; (2) bloodstream infection;(3) direct spread of intra-abdominal infection; (4) direct invasion; and (5) cryptogenic infection.

As can be seen from these routes of invasion, the pathogens mainly come from digestive system and abdominal cavity. While Klebsiella pneumoniae is the main pathogen in Asia (4), Escherichia coli, K. pneumoniae, and Streptococcus are the main pathogens in western countries (5). In addition, Anaerobes and Staphylococcus aureus can also cause liver abscess. Fusobacterium nucleatum is a Gramnegative obligate anaerobe that mainly colonizes the oral cavity and is considered an opportunistic pathogen. Liver abscesses caused by F. nucleatum are very rare, and our case is reported as the first case in China.

\section{Case Presentation}

The patient was a 34-year-old female. She had fever with highest temperature of $40.2^{\circ} \mathrm{C}$ without obvious inducement. The patient was immunocompetent and had a good oral hygiene. There was no history of using drugs associated with reduced immunity, such as hormones or chemotherapy. No diseases were found in oral and digestive tract examination. White blood cell count was 8.21 $\times 10^{9} / \mathrm{L}$, neutrophil count was $83.8 \%$, high sensitivity Creactive protein (CRP) was $95.6 \mathrm{mg} / \mathrm{L}$, and procalcitonin (PCT) was $0.22 \mathrm{ng} / \mathrm{mL}$. Abdominal computed tomography (CT) scan showed multiple small patchy hypodense foci in the right lobe of the liver (Figure $1 \mathrm{~A}$ ).

Abdomen magnetic resonance imaging (MRI) showed multiple masses of abnormal signal shadow of the liver. The larger one was located in the liver with the right upper lobe, and its size was about $74 \times 47 \mathrm{~mm}$ (Figure $1 \mathrm{~B}$ ). Considering that the patient may have liver abscess, liver biopsy was performed. The results showed that the liver tissue was inflammatory, and the diagnosis of liver abscess was clear. The patient was given puncture and drainage of liver abscess. However, drainage was not effective, and only a little bloody secretion was drained. So, the patient was 
given empirical anti-infective treatment. cefoperazone sulbactam was used; but since the body temperature still fluctuated, we changed the treatment to imipenem $1 \mathrm{~g}$ q8h intravenous drip after 5 days.

After anti-infective treatment, the inflammatory index of patients decreased slightly, and the patient's temperature dropped to $37.7^{\circ} \mathrm{C}$. The white blood cell count was $10.62 \times 10^{9} / \mathrm{L}$, neutrophil count was $8.7 \times 10^{9} / \mathrm{L}$, CRP was $76.1 \mathrm{mg} / \mathrm{L}$, and PCT was $0.10 \mathrm{ng} / \mathrm{mL}$. In order to further identify the pathogen and adjust the use of antibiotics, liver abscess puncture was performed under ultrasound guidance and $4 \mathrm{~mL}$ of pus was aspirated. Since the clinical laboratory center had not cultured pathogens, the pus was sent to a third-party laboratory for microbiological examination by genetic detection. Next-generation sequencing (NGS) results showed that the pathogen was F. nucleatum (Sequences Number \# 43). Based on the results of NGS, we adjusted the medication regimen. Imipenem was discontinued after 8 days of use and changed to metronidazole 80 $\mathrm{mL}$ q6h, amoxicillin sodium, and clavulanate potassium 1.2 g q8h.

After targeted anti-infective treatment, the patient's temperature dropped to $36.3^{\circ} \mathrm{C}$. The white blood cell count was $6.43 \times 10^{9} / \mathrm{L}$, and CRP was $11.2 \mathrm{mg} / \mathrm{L}$. The inflammatory index decreased significantly. Abdominal CT showed that the lesions were significantly reduced (Figure 2), and antiinfective therapy was effective. There were no other complications in the whole course of the disease. After 20 days of treatment, the patient recovered and discharged. After discharge, the patient took amoxicillin clavulanate tablets $312.5 \mathrm{mg}$ orally three times a day as well as metronidazole tablets q6h $0.4 \mathrm{~g}$ orally. One month later, the follow-up showed that the patient's temperature was normal.

\section{Discussion}

Pyogenic liver abscess is a common infectious disease of the liver, and early diagnosis is difficult. Severe sepsis, septic shock, and other serious life-threatening diseases could make the treatment time longer. In recent years, the diagnosis and treatment of the disease became difficult due to the following reasons: the increase of diabetes, malignant tumor, biliary tract disease, and abdominal infectious diseases; the production of multiple drug-resistant and highly virulent pathogens; and the increased number of interventional operations in the digestive system. There are many ways for pathogens to invade the liver and lead to liver abscess. Biliary tract infection is the most common cause of liver abscess, followed by intestinal or pelvic diseases $(6,7)$. Because of its route of transmission, pathogens mostly come from the digestive system and abdominal cavity. The main pathogenic bacteria of liver abscess in western countries are E. coli, K. pneumoniae, Enterococcus, and Streptococcus (5). In Asia, K. pneumoniae is the main pathogen. A study showed that K. pneumoniae is the main infection in the east region in China (4).

Pyogenic liver abscess caused by $F$. nucleatum is extremely rare, and as far as we investigated, there is no relevant case report in China. F. nucleatum is a common Gramnegative obligate anaerobe that mainly colonizes the human oral cavity and digestive tract. It can lead to brain abscess, appendicitis, pelvic inflammatory disease, etc. $(8,9)$. There are related cases reported that liver abscess caused by F. nucleatum may be due to poor oral hygiene (10) or oral diseases $(11,12)$. Digestive system diseases such as sigmoid diverticulitis (13) and abdominal scar (14) may also be the cause of liver abscess caused by F. nucleatum. In our case, the patient had a good oral hygiene and there were no digestive system diseases. So, there was no typical pathogenic cause.

Diagnosis of $F$. nucleatum infection usually requires culture of the pathogen. However, due to the strict anaerobic environment required for culture and the high requirement for specimen collection, sometimes the pathogens could not be cultured. In a case report by Jayasimhan et al., they did not culture F. nucleatum from pus, but found it through blood culture (15). They considered that the delay in pus sampling and early anti-microbial treatment could affect the culture results. In this case, there was also no pathogen cultured from the pus, which made the further diagnosis and treatment of the disease difficult. Through NGS, the pathogen was finally identified as F. nucleatum.

In NGS, also known as high-throughput sequencing, metagenomic NGS is commonly used in microbial detection. As there is currently no uniform standard in the clinic, it has not become the recommended test, but we can use its advantages to solve practical problems. In 2014, a laboratory in the United States successfully used the NGS to detect Leptospira from a 14-year-old boy's cerebrospinal fluid, and timely anti-infective treatment saved his life. Five months later, the Centers for Disease Control and Prevention (CDC) confirmed their diagnosis by serological method (16). In this case, because the pathogen could not be cultured, NGS of pus was used to identify the pathogen, which provided an important basis for further targeted anti-infective treatment. We believe that the failure to culture the pathogen may be related to empirical medication during the initial stage of treatment. The use 

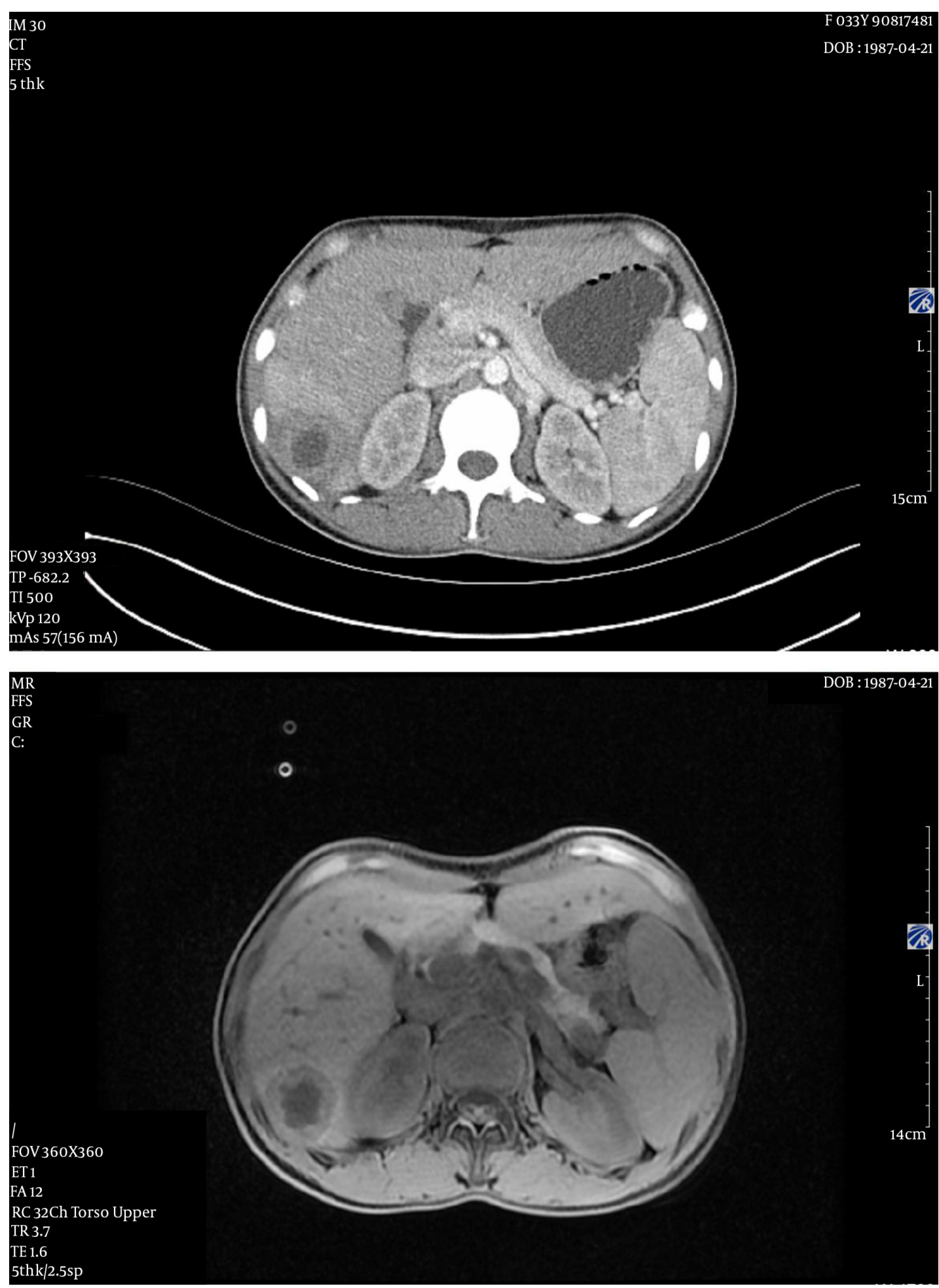

Figure 1. A, abdominal CT; and B, MRI indicating an abscess in the right lobe of the liver. 


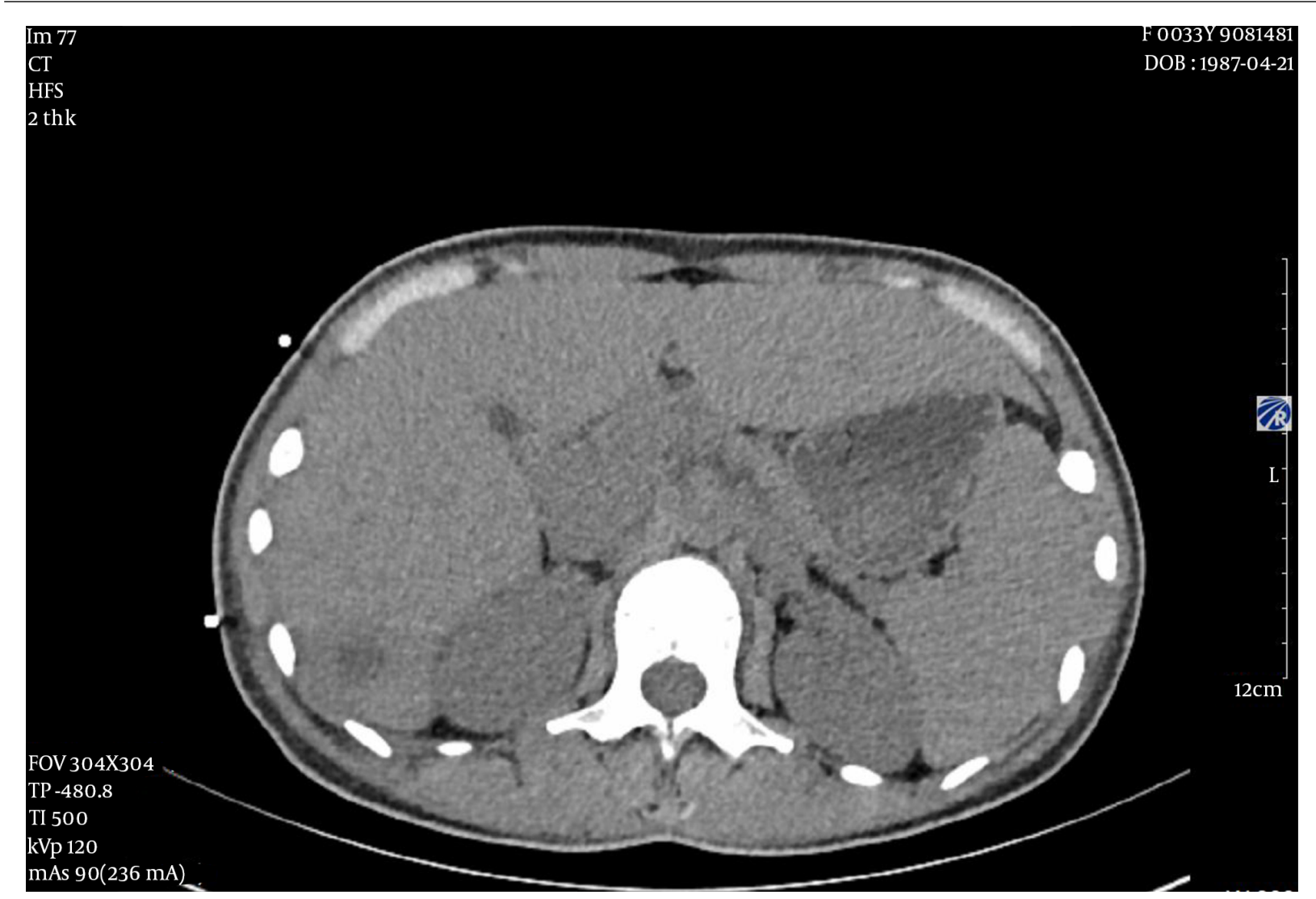

Figure 2. Abdominal CT indicating significantly reduced lesions

of antibiotics in early treatment inhibited the pathogenic bacteria. NGS results showed that the relative abundance of $F$. nucleatum was not high, which confirmed our inference.

\subsection{Conclusion}

Our case report is valuable mainly for these reasons. Firstly, this is the first case of liver abscess caused by $F$. nucleatum in China. Secondly, in this case, we identified the pathogen and successfully cured the patient without culturing pathogenic bacteria. The idea of diagnosis and treatment can be used as a reference. Thirdly, this case reminds us that in order to improve the positive rate of anaerobic bacteria culture, we should pay attention to avoid contacting with air in the process of specimen collection when the pathogenic bacteria are suspected to be anaerobic bacteria. Finally, as a new microbial detection method, NGS can still be helpful in clinical practice, although it has not been widely used.

\section{Footnotes}

Authors' Contribution: WWH, FWJ, and ZZW collected and analyzed the data, and conceived the case report. XCA and WSH collected the data and participated in the design of the case report. TCH, LX, and PHY analyzed the data and drafted the manuscript. All the authors contributed to the clinical care of the patient and gave final approval of the manuscript to be published.

Conflict of Interests: The authors declare that they have no competing interests.

Ethical Approval: This study has been reviewed and approved by the Research Ethics Committee of Zhejiang Provincial Peoples Hospital (ref\#2021QT195).

Funding/Support: No funds have been provided for this study.

Informed Consent: Written informed consent was obtained from the patient. 


\section{References}

1. Tsai FC, Huang YT, Chang LY, Wang JT. Pyogenic liver abscess as endemic disease, Taiwan. Emerg Infect Dis. 2008;14(10):1592-600. doi: 10.3201/eid1410.071254. [PubMed: 18826824]. [PubMed Central: PMC2609891].

2. Meddings L, Myers RP, Hubbard J, Shaheen AA, Laupland KB, Dixon E, et al. A population-based study of pyogenic liver abscesses in the United States: Incidence, mortality, and temporal trends. Am J Gastroenterol. 2010;105(1):117-24. doi: 10.1038/ajg.2009.614. [PubMed: 19888200].

3. Kuo SH, Lee YT, Li CR, Tseng CJ, Chao WN, Wang PH, et al. Mortality in Emergency Department Sepsis score as a prognostic indicator in patients with pyogenic liver abscess. Am J Emerg Med. 2013;31(6):916-21. doi:10.1016/j.ajem.2013.02.045. [PubMed: 23623237].

4. Qu TT, Zhou JC, Jiang Y, Shi KR, Li B, Shen P, et al. Clinical and microbiological characteristics of Klebsiella pneumoniae liver abscess in East China. BMC Infect Dis. 2015;15:161. doi: 10.1186/s12879-015-0899-7. [PubMed: 25886859]. [PubMed Central: PMC4381403].

5. Petri A, Hohn J, Hodi Z, Wolfard A, Balogh A. Pyogenic liver abscess 20 years' experience. Comparison of results of treatment in two periods. Langenbecks Arch Surg. 2002;387(1):27-31. doi:10.1007/s00423-0020279-9. [PubMed: 11981681].

6. Rahimian J, Wilson T, Oram V, Holzman RS. Pyogenic liver abscess: Recent trends in etiology and mortality. Clin Infect Dis. 2004;39(11):16549. doi: 10.1086/425616. [PubMed: 15578367].

7. Heneghan HM, Healy NA, Martin ST, Ryan RS, Nolan N, Traynor O, et al. Modern management of pyogenic hepatic abscess: A case series and review of the literature. BMC Res Notes. 2011;4:80. doi: 10.1186/17560500-4-80. [PubMed: 21435221]. [PubMed Central: PMC3073909].

8. Han XY, Weinberg JS, Prabhu SS, Hassenbusch SJ, Fuller GN, Tarrand JJ, et al. Fusobacterial brain abscess: A review of five cases and an analysis of possible pathogenesis. J Neurosurg. 2003;99(4):693-700. doi: 10.3171/jns.2003.99.4.0693. [PubMed: 14567605].
9. Swidsinski A, Dorffel Y, Loening-Baucke V, Theissig F, Ruckert JC, Ismail $\mathrm{M}$, et al. Acute appendicitis is characterised by local invasion with Fusobacterium nucleatum/necrophorum. Gut. 2011;60(1):34-40. doi: 10.1136/gut.2009.191320. [PubMed: 19926616].

10. Nagpal SJ, Mukhija D, Patel P. Fusobacterium nucleatum: A rare cause of pyogenic liver abscess. Springerplus. 2015;4:283. doi:10.1186/s40064015-1090-8. [PubMed: 26101735]. [PubMed Central: PMC4472653].

11. Collins L, Diamond T. Fusobacterium nucleatum causing a pyogenic liver abscess: A rare complication of periodontal disease that occurred during the COVID-19 pandemic. BMJ Case Rep. 2021;14(1). doi: 10.1136/bcr-2020-240080. [PubMed: 33500312]. [PubMed Central: PMC7843314].

12. Hammami MB, Noonan EM, Chhaparia A, Khatib FA, Bassuner J, Hachem C. Denture-associated oral microbiome and periodontal disease causing an anaerobic pyogenic liver abscess in an immunocompetent patient: A case report and review of the literature. Gastroenterology Res. 2018;11(3):241-6. doi: 10.14740/gr1006w. [PubMed: 29915637]. [PubMed Central: PMC5997471].

13. Wijarnpreecha K, Yuklyaeva N, Sornprom S, Hyman C. Fusobacterium nucleatum: Atypical organism of pyogenic liver abscess might be related to sigmoid diverticulitis. N Am J Med Sci. 2016;8(4):197-9. doi: 10.4103/1947-2714.179961. [PubMed: 27213146]. [PubMed Central: PMC4866478].

14. Inoue K, Watanabe $Y$, Nagata H, Kojima M. Pyogenic liver abscess caused by Fusobacterium nucleatum with an inflammatory scar in the abdominal cavity. Clin J Gastroenterol. 2021;14(2):617-20. doi: 10.1007/s12328-021-01376-9. [PubMed: 33675513].

15. Jayasimhan D, Wu L, Huggan P. Fusobacterial liver abscess: A case report and review of the literature. BMC Infect Dis. 2017;17(1):440. doi: 10.1186/s12879-017-2548-9. [PubMed: 28633639]. [PubMed Central: PMC5477746].

16. Wilson MR, Naccache SN, Samayoa E, Biagtan M, Bashir H, Yu G, et al. Actionable diagnosis of neuroleptospirosis by next-generation sequencing. $N$ Engl J Med. 2014;370(25):2408-17. doi: 10.1056/NEJMoa1401268. [PubMed: 24896819]. [PubMed Central: PMC4134948]. 